\title{
Immunohistochemical Expression of Collagen IV and MMP9 in Odontogenic Cysts and Tumors
}

\author{
Homeyra Mardani, Nastaran Dinarvand*, \\ Gholamreza Jahanshahi and Elaheh Sofiyan
}

Department of Oral and Maxillofacial Pathology, School of Dentistry, University of Medical Sciences, Isfahan (Khorasgan) Branch, Islamic Azad University, Isfahan, Iran.

DOI: http://dx.doi.org/10.13005/bbra/1911

(Received: 09 July 2015; accepted: 21 August 2015)

\begin{abstract}
The growth mechanism of odontogenic cysts and tumors along with their invasion and destructive ability can be influenced by the secretion of metalloproteinases from epithelial and mesenchymal cells. An important activity of matrix metalloproteinases is destruction of the components of basement membrane, including collagen IV. The present study was conducted to analyze the role of collagen IV and its association with matrix metalloproteinases9 in odontogenic cysts and tumors. In this study, 19 samples of keratocyst odontogenic tumor and 11 samples of dentigerous cyst in mesenchymal stroma and odontogenic epithelium, and in basement membrane of odontogenic epithelial neoplastic islands and basement membrane of cystic epithelium were analyzed by immunohistochemical method. Collagen IV expression in keratocyst odontogenic tumors and dentigerous cysts was $>\mathbf{5 0} \%$, which showed no statistically significant difference $(p=0.62)$. The consistency and thickness of collagen IV in dentigerous cysts were significantly higher than those of keratocyst odontogenic tumors in terms of the staining pattern of collagen IV $(p=0.003, p=0.001)$. Also, no significant difference was observed between the epithelium of keratocyst odontogenic tumors and dentigerous cysts with regard to the expression of matrix metalloproteinase-9 $(p=0.18)$. Expression of metalloproteinases9 in the mesenchymal stroma of keratocyst odontogenic tumors was significantly higher than dentigerous cysts $(p=0.04)$. Higher expression of matrix metalloproteinase-9 and inconsistency and low thickness of collagen IV in keratocyst odontogenic tumors may be associated with diverse biological behavior of keratocyst odontogenic tumors than dentigerous cysts.
\end{abstract}

Key words: Keratocystic odontogenic tumor, dentigerous cyst, matrix metalloproteinase9, collagen IV, immunohistochemical .

Odontogenic cysts and tumors constitute a heterogeneous group of jaw lesions with diverse histopathological features and clinical expression. 1 Keratocystic odontogenic cyst has currently been classified as Keratocystic odontogenic tumor (KOT) in new classification owing to more invasive behavior and higher recurrence rate than odontogenic cysts ${ }^{1-2}$. In contrast, dentigerous cyst(DC) has a mild behavior and rarely recurs

\footnotetext{
* To whom all correspondence should be addressed.
}

after removal. Keratocystic odontogenic tumor (KOT) has a cystic structure similar to dentigerous cyst, but it has an aggressive development pattern similar to ameloblasts ${ }^{3}$.

Given the varied behavior of these lesions, a number of studies have been carried out to analyze the epithelial and mesenchymal factors that determine the different behaviors of these lesions. Some events, the same as histomorphological differentiation of tooth development in odontogenic lesions, occur during tumor development and mesenchymal-epithelial interaction. 
Most of the studies have been conducted on the epithelial components of tumors and odontogenic cysts ${ }^{4}$. The development mechanism of odontogenic cysts and invasive and destructive capacity of some odontogenic tumors may be influenced by matrix metalloproteinases, secreted from epithelial and mesenchymal cells ${ }^{5-6}$.

Changes in the expression of specific proteins of extracellular matrix (ECM) with increasing presence of matrix metalloproteinases and lack of expression of metallopeptidase inhibitors (TIMPs) may influence the behavior of these lesions and consequently affect the development and aggressiveness of tumors ${ }^{7}$.

Matrix metalloproteinases are the most important enzymes, responsible for disintegration and destruction of extracellular matrix (ECM), whose major function is removal of the molecules in extracellular matrix.8 Matrix metalloproteinases are a family of calcium- and zinc-dependent endopeptidases that are classified based on the molecular structure and the substance they destroy. Matrix metalloproteinases are divided into two groups of soluble matrix metalloproteinases and membrane-related matrix metalloproteinase. The soluble types include collagenases, gelatinases, stromelysines, matrilizines and a heterogeneousgroup 9 .

From among matrix metalloproteinases, matrix metalloproteinase9 is the most important proteinase associated with bone resorption, whose increase in tissues causes the destruction and breakage of extracellular matrix molecules and molecules on the cell surface, thereby changing the cell-cell and cell-extracellular matrix interaction ${ }^{10}$. Matrix metalloproteinases can also aim to destroy molecules other than extracellular matrix components ${ }^{11-12}$. However, recent studies have shown that matrix metalloproteinases cannot explain the aggressive behaviors of the mentioned lesions alone, and their interaction with other factors such as collagen IV can affect their biological behaviors.

Collagen IV is one of the components of basement membrane (BM) that is involved in all stages of tooth development. Further, changes and remodeling of basement membrane collagen IV occur in most of the odontogenic neoplasms ${ }^{13}$. Collagen IV along the basement membrane also plays a pivotal role in cell attachment, migration, differentiation and development, and its reduced production during angiogenesis processes also plays an important role in tissue remodeling and tumor growth ${ }^{14}$.

Few studies have investigated the role of above factors in odontogenic cysts and tumors as well as their biologic behaviors. Hence, for better understanding of biologic behaviors of odontogenic lesions, extracellular matrix assessment is required in order to determine different expression levels of proteins among indolent and invasive lesions ${ }^{15}$. Therefore, the present study was performed to investigate the immunohistochemical expression of matrix metalloproteinase ${ }^{9}$ and collagen IV in keratocystic odontogenic tumors and dentigerous cysts to better understand the association of matrix metalloproteinase ${ }^{9}$ with destruction of basement membrane and biologic behavior of these lesions.

\section{MATERIALSAND METHODS}

In this retrospective descriptiveanalytical research, the odontogenic cysts including keratocystic odontogenic tumors and dentigerous cysts were studied. This study (research project number:2381020921038) was approved by the ethics committee and vice chancellery of research at Khorasegan branch, Islamic Azad University, Isfahan and conducted over one year, 2013-2014.

The blocks of 30 odontogenic cysts, 19 keratocystic odontogenic tumors and 11 dentigerous cysts were obtained from the pathology department of Isfahan school of dentistry. Clinical information, including age, gender and lesion site was analyzed. A $5 \mu$ section was prepared for hematoxylin-eosin staining. Having observed hematoxylin-eosin slides, the slides with sufficient tissue and appropriate fixation were selected. The samples with improper fixation, extensive inflammation, extensive areas of bleeding and too much inflammation, Gorlin-syndromerelated odontogenic keratocysts and odontogenic lesions with inadequate tissue were excluded from the study. Diagnosis of odontogenic cysts and tumors was performed according to the special classification of World Health Organization (WHO) in 2005 and other studies ${ }^{16-18}$. The expression levels of collagen IV and matrix metalloproteinase-9 were 
determined and compared in both groups.

\section{Immunohistochemical methods}

Immunohistochemical staining was performed by envision method using matrix metalloproteinase-9 monoclonal antibody (Biogenex co, USA) and collagen IV monoclonal antibody (Dako co, USA). $3 \mu$ sections prepared from the blocks were attached on the glass slides. During deparaffinization and dehydration processes, the slides were put in thermal furnace $\left(60^{\circ} \mathrm{C}\right)$ for 30 minutes and then placed in two xylol containers for 5 minutes and three descending ethanol (100\%, $80 \%$ and $65 \%$, respectively) containers for 2 minutes in order to dehydrate. The slides were then placed in 3\% oxygenated water and washed with distilled water after 5 minutes.

During antigen retrieval stage for matrix metalloproteinase-9 marker, slides were placed in Tris-EDTA buffer $(\mathrm{pH}=9)$ in microwave for 20 minutes. For collagen IV marker, slides were put in proteinase $\mathrm{K}$ for 20 minutes. Then, the primary antibodies of matrix metalloproteinase-9 and collagen IV were separately poured on the tissue. After 60 minutes incubation for collagen IV and 30 minutes incubation for matrix metalloproteinase- 9 at room temperature, the slides were washed with phosphate buffered saline.

In chromogen stage, the slideswere stained by diaminobenzidine chromogen for 5 minutes and then washed with distilled water. Omission of primary antibody was employed as negative control, while tissue of long and skin were used as positive control for MMP9 and collagen IV. Brown cytoplasmic staining was considered as positive. The histological regions in which the given marker was expressed were observed as brown color under optic microscope. Hematoxylin staining was used for background stating. Finally, the slides were mounted with entelan glue and observed under optic microscope.

Having stained the slides, they were evaluated by two pathologists under optic microscope. According to the methods presented by Gomes Henriquesg in 2011, for collagen IV staining, the basement membrane between epithelium and mesenchyme was analyzed. The percentage of collagen IV staining along the basement membrane was analyzed semiquantitatively as $>50 \%$ or d" $50 \%$. Also, the staining pattern of collagen was determined in terms of consistency (continuous, discontinuous) and thickness (thin, thick and mixed).19Expression of matrix metalloproteinase-9 was separately analyzed in epithelial and mesenchymal lesions. In mesenchyme, staining was observed in endothelial cells, fibroblasts and inflammatory cells. The staining percentage of matrix metalloproteinase-9 was determined semi-quantitatively using the method presented by Gong 20 in 2009. In this method, the staining percentage is determined by one of these three scores, 0 ( $<10 \%$ staining), 1 (10$50 \%$ staining) and 2 (>50\% staining). Brown cytoplasm was considered as positive staining.

\section{Statistical analysis}

The obtained data were analyzed by SPSS-20 software using chi-square, MannWhitney and Fisher's exact tests.

\section{RESULTS}

A total of 19 keratocystic odontogenic tumors of the patients with age range of $15-70$ years and mean age of $16.40 \pm 33.63$ and 11 dentigerous cysts of the patients with age range of 7-55 and mean age of $17.56 \pm 29.81$ were analyzed in this study. With regard to the lesion site, from the 19 keratocystic odontogenic tumors, 14 samples were related to posterior mandible and 5 samples were related to maxilla. Of 11 dentigerous cysts, 5 pericoronal samples were found in impacted mandibular wisdom tooth, 1 pericoronal sample in mandibular tooth 5,1 pericoronal sample in maxillary canine and 4 samples in intraosseous area (3 samples in anterior maxilla and 1 sample in posterior mandible).

From the samples studied in OKC group, $64.3 \%$ were male and $31.6 \%$ were female. As for the dentigerous cyst group, $63.6 \%$ of samples were male and $36.4 \%$ were female. The distribution of gender was not significantly different between groups ( $\mathrm{p}=0.79$ ). The expression levels of matrix metalloproteinase- 9 in the epithelial and mesenchymal components of keratocystic odontogenic tumors and dentigerous cysts are presented in Table 1and Figure4,5,6

The expression levels of collagen IV in the basement membrane and staining pattern of collagen in terms of consistency (continuousdiscontinuous) in keratocystic odontogenic tumors and dentigerous cysts are presented in Table 2. 
Table 1. Immunohistochemical expression of matrix metalloproteinase-9 in the epithelial and mesenchymal components of keratocystic odontogenic tumors and dentigerous cysts

\begin{tabular}{lccc}
\hline $\begin{array}{l}\text { Immunohistochemical } \\
\text { expression of MMP9 }\end{array}$ & $\begin{array}{c}\text { Keratocystic } \\
\text { odontogenic tumor } \\
\mathrm{n}=19\end{array}$ & $\begin{array}{c}\text { Dentigerous } \\
\text { cysts } \\
\mathrm{n}=11\end{array}$ & P value \\
\hline Epithelium[n(\%)] & $7(36.8 \%)$ & $5(45.5 \%)$ & \\
-Score0 & $12(63.2 \%)$ & $6(54.5 \%)$ & 0.18 \\
-Score1 & $0(0 \%)$ & $0(0 \%)$ & \\
-Score2 & & & \\
Mesenchyme [n(\%)] & $3(15.8 \%)$ & $3(27.3 \%)$ & 0.04 \\
-Score0 & $12(63.2 \%)$ & $8(72.7 \%)$ & \\
-Score1 & $4(21.2 \%)$ & $0(0 \%)$ & \\
-Score2 & &
\end{tabular}

Table 2.Expression of collagen IV and its patterns (continues or discontinues) at the basement membrane of keratocystic odontogenic tumors and dentigerous cysts

\begin{tabular}{lccc}
\hline & $\begin{array}{c}\text { Keratocystic odontogenic } \\
\text { tumor } \mathrm{n}=19\end{array}$ & $\begin{array}{c}\text { Dentigerous cysts } \\
\mathrm{n}=11\end{array}$ & P value \\
\hline $\begin{array}{l}\text { Expression of collagen IV } \\
-<50 \% \text { staining }\end{array}$ & $4(21.1 \%)$ & $2(18.2 \%)$ & 0.62 \\
$->50 \%$ staining & $15(78.9 \%)$ & $9(81.8 \%)$ & \\
Staining distribution patterns & & & 0.003 \\
- Continuous & $5(26.3 \%)$ & $9(81.8 \%)$ & \\
- Discontinuous & $14(73.7 \%)$ & $2(18.2 \%)$ & \\
\hline
\end{tabular}

The staining patterns of collagen with regard to thickness (thin, thick and mixed) in keratocystic odontogenic tumors and dentigerous cysts are presented in Figure 1,2, 3. The thickness of collagen
IV in the basement membrane of dentigerous cyst was significantly higher than that of keratocystic odontogenic tumor $(\mathrm{p}=0.001)$.

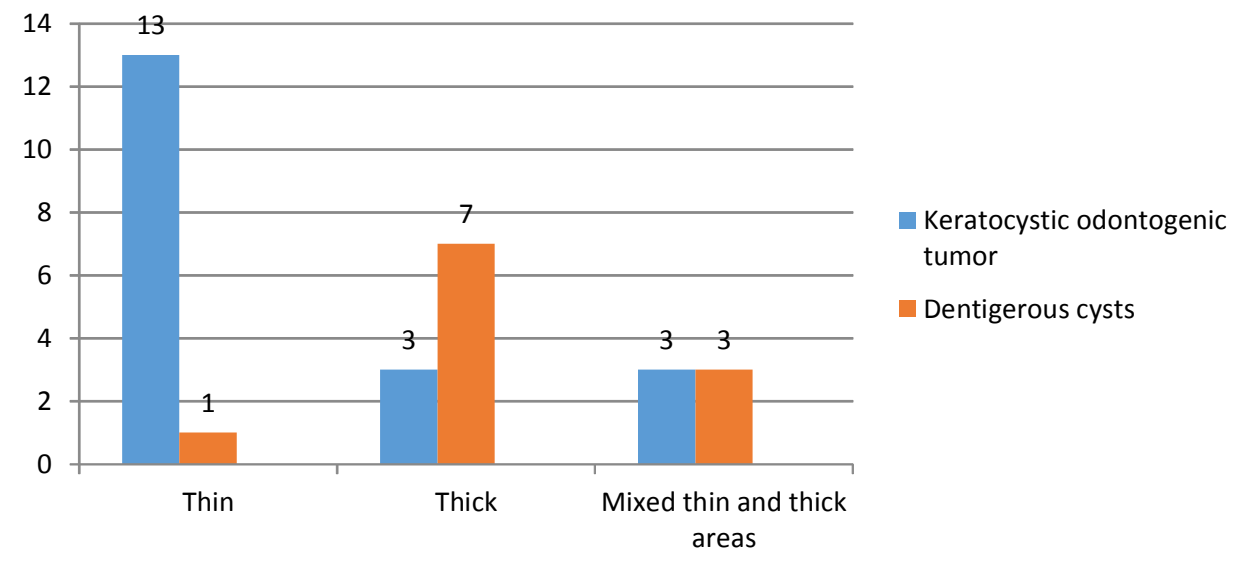

Fig. 1. The staining patterns of collagen IV expression according to thickness (thin, thick and mixed thin and thick areas) at the basement membrane of keratocystic odontogenic tumors and dentigerous cysts 


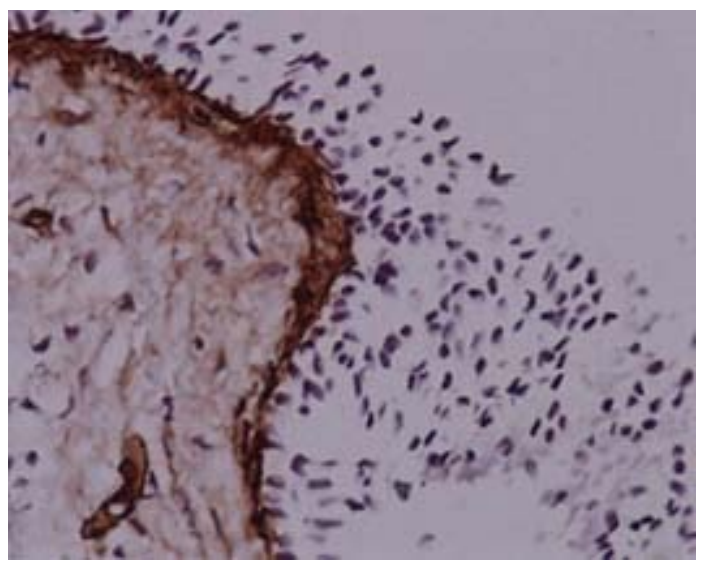

Fig. 2. Thick basement membrane in dentigerous cyst $(\times 400)$

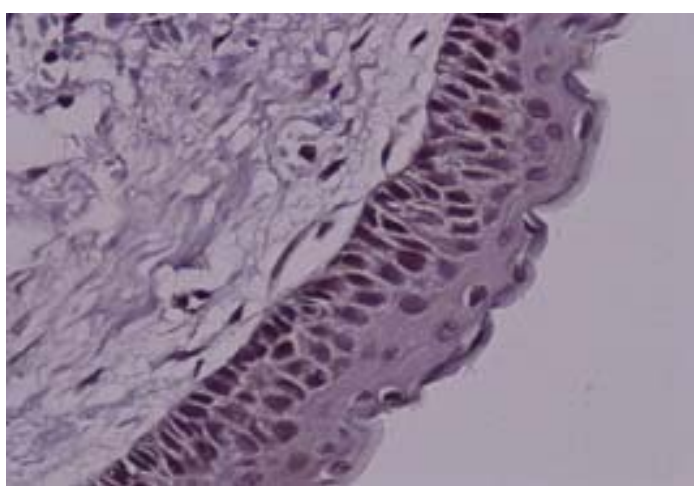

Fig. 4. Score 1 in the epithelial keratocystic odontogenic tumor $(\times 400)$

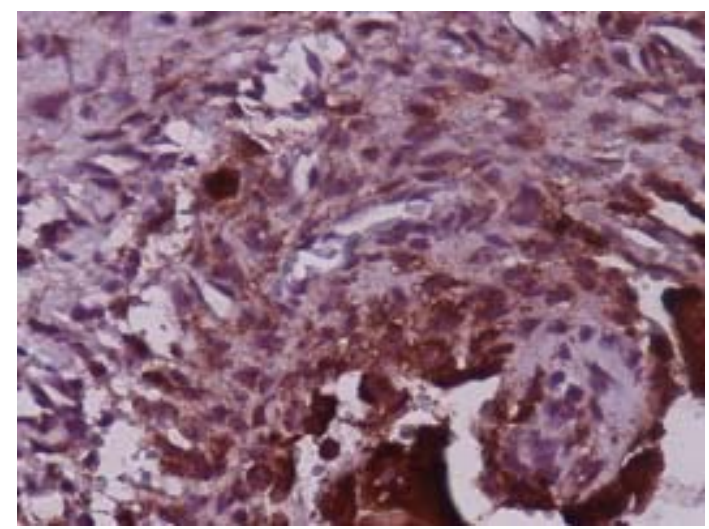

Fig. 6. Score 2 in the mesenchymal keratocystic odontogenic tumor $(\times 400)$

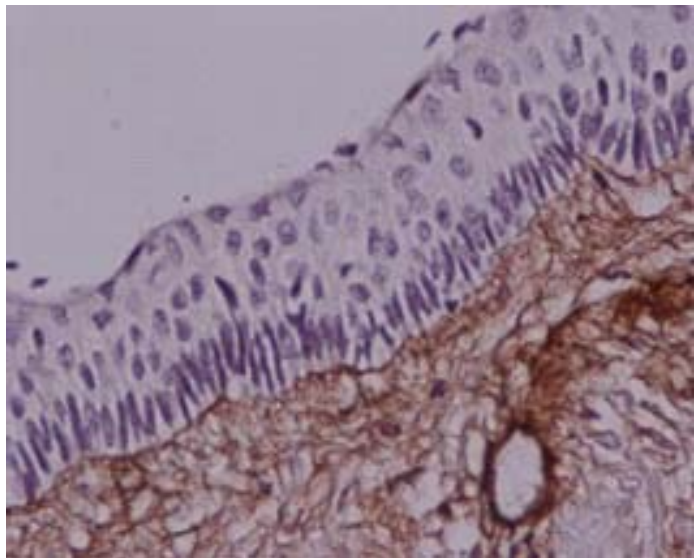

Fig. 3. Thin basement membrane in keratocystic odontogenic tumor $(\times 400)$

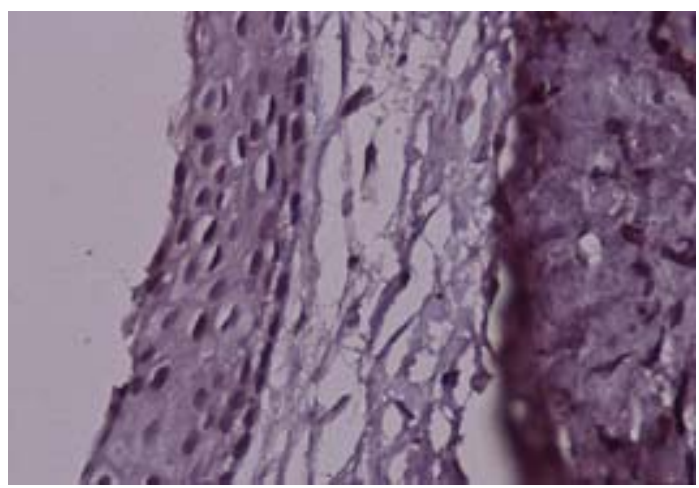

Fig. 5. Score 0 in Mesenchymal dentigerous cyst $(\times 400)$

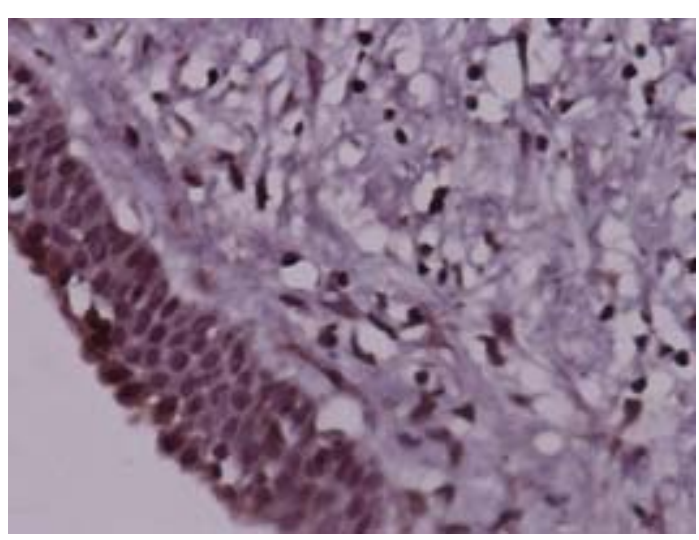

Fig. 7. Score 1 in the epithelial and mesenchymal keratocystic odontogenic tumor $(\times 400)$ 


\section{DISCUSSION}

In this study we investigated the immunohistochemical expression of matrix metalloproteinase- 9 and collagen IV in keratocystic odontogenic tumors and dentigerous cysts. The results indicated that immunohistochemical expression of matrix metalloproteinase-9 in the epithelial component of odontogenic tumors and cysts was not significantly different, but its expression was significantly higher in keratocystic odontogenic tumors than in dentigerous cysts. Expression of collagen IV was similar in the two studied pathologies. Continuous staining patterns were more frequent in dentigerous cysts, whereas discontinuous patterns were more prevalent in odontogenic tumors. Expression of collagen IV was significantly thicker in dentigerous cysts.

Several studies have investigated the role of matrix metalloproteinases in the growth of odontogenic tumors and cysts. Recently, Scariot et al 21 systematically reviewed the role of matrix metalloproteinases in this group of lesions and reported that the expression patterns of matrix metalloproteinases in odontogenic lesions were different from the expression patterns of other lesions. Accordingly, odontogenic lesions especially keratocystic odontogenic tumors showed a significantly positive immunoreactivity against matrix metalloproteinases. They suggested that matrix metalloproteinases could explain the aggressive biological behavior of these tumors. According to their findings, matrix metalloproteinases could degrade some types of collagen, such as collagen type IV and basement membrane laminin. They presented on the walls of odontogenic cysts and in the cystic fluid of these dental lesions. There is evidence regarding the involvement of matrix metalloproteinase- 9 in the pathologic process of cyst expansion. Moreover, matrix metalloproteinase-9 is secreted in inactive form, and it should convert to its active form to represent its function.

Some previous studies have demonstrated a positive association between matrix metalloproteinase- 9 activity and the type of odontogenic cysts ${ }^{22}$. Given the association between matrix metalloproteinase- 9 and collagen IV as well as the recent reports regarding the interaction of matrix metalloproteinases with other unknown factors for biological behavior of odontogenic tumors and cysts, we designed this study and compared the expression of both matrix metalloproteinase- 9 and collagen IV in keratocystic odontogenic tumors and dentigerous cysts ${ }^{23}$.

The structure of basement membrane, especially extracellular matrix plays an important role in regulating the cellular behavior. The role of basement membrane of vessels in regulation of angiogenesis is well-known and is used for the treatment of cancers ${ }^{24}$. The origin of basement membrane collagen IV is still unknown, whether from epithelial or mesenchymal cells.

Several studies have indicated the immunohistochemical expression of $\alpha 3, \alpha 4, \alpha 5$ and $\alpha 6$ chains of collagen IV in normal tissues that are expressed based on the epithelial type of collagen IV.25,26,27 Many studies have shown that $\alpha$ chains of collagen IV are destroyed at the initial stages of invasion in many cancers ${ }^{27,29,30,31,32}$.

The initial stages of invasion and metastasis of cancer cells occur as a result of destruction of basement membrane, which in turn happens through the effect of matrix metalloproteinases that are produced from cancer cells and reduction in inhibitors of matrix metalloproteinases. Apart from the aforementioned mechanism, some studies have shown that hypermethylation of collagen chains in COL4A6 and COL4A5 can account for the lost expression of $\alpha 5$ (IV)/ $\alpha 6$ (IV) chains and remodeling of basement membrane during the invasion of cancer cells ${ }^{33}$.

Modification of basement membrane collagen IV has also been observed in odontogenic neoplastic lesions ${ }^{13}$. In the current study, the expression of collagen IV was not reported to be different between keratocystic odontogenic tumors and dentigerous cysts, but the staining pattern and collagen thickness were different in these lesions. Lack of difference between collagen IV in odontogenic tumors and cysts can be associated with hypermethylation of collagen chains. Since reduced expression of $\alpha 5$ (IV)/ $\alpha 6$ (IV) chains have been seen in many cancers, further studies are required to analyze odontogenic lesions in terms of hypermethylation and modification of basement membrane.

In normal and benign mucosa, collagen IV is expressed as a continuous and thick layer 
with high staining capacity ${ }^{34-35}$. However, the results of studies by Gomes Henriques and Oliveira indicated continuous and thick expression of collagen IV in odontogenic lesions, and radicular and dentigerous cysts, and discontinuous and thin expression of collagen IV in keratocystic odontogenic tumors ${ }^{4,19}$. The results for the staining pattern of collagen IV in dentigerous cysts and keratocystic odontogenic tumors in the present study were in line with those of the studies by Henriques, except that in the current study the staining intensity in dentigerous cysts and keratocystic odontogenic tumors was similar (both $>50 \%-50 \%$ ), with no significant difference between them.

Gomes Henriques et al conducted a study in 2011 and reported that the staining level in collagen IV was basically $>50 \%$ in dentigerous cysts and $<50 \%$ in keratocystic odontogenic tumors. They also showed that the staining pattern of collagen IV was continuous and thick in dentigerous cysts and discontinuous and thin in keratocystic odontogenic tumors ${ }^{19}$.

In the present study, the expression of collagen IV was $>50 \%$ in $78.9 \%$ of keratocystic odontogenic tumors and $81.8 \%$ of dentigerous cysts. In most keratocystic odontogenic tumors, collagen IV showed a discontinuous staining pattern and was observed as a thin layer; whereas in most dentigerous cysts, collagen IV was observed as a continuous and thick layer all over the basement membrane. However, the findings of Gomes are different than our results in terms of the expression level of collagen IV, which can be associated with different immunohistochemical techniques used. The results of Gomes et al's study are in agreement with the findings of the present research with regard to the thickness and consistency of collagen in keratocystic odontogenic tumors and dentigerous cysts.

Unlike the results of studies regarding the correlation of continuity and consistency of basement membrane with aggressive activity of lesions, Poomsawat et al reported no significant correlation in the expression levels of components of basement membrane (collagen IV, laminin 1 and 5, and fibronectin) among dentigerous cysts, keratocystic odontogenic cysts and radicular cysts. These results are in contrast with our findings, which can be due to the different immunohisto chemical techniques used in their study and ours ${ }^{36}$. As for the expression of matrix metalloproteinase-9, most of the studies have shown a significantly higher expression of matrix metalloproteinase-9 in the mesenchyme of keratocystic odontogenic cysts than dentigerous and radicular cysts. This can be a reason for more aggressive behavior and higher potential for development of keratocystic odontogenic tumors than other odontogenic cysts.

Silveira et al performed a study in 2007 and showed that the growth and development mechanism of keratocystic odontogenic tumors, and dentigerous and radicular cysts can be influenced by the development and secretion of matrix metalloproteinases ${ }^{5}$.

In addition, the findings of Gomes Henriques indicated that the expression of matrix metalloproteinase-9 in the epithelium of keratocystic odontogenic tumor, dentigerous cyst, radicular cyst and ameloblastoma showed no significant difference, but its expression in the mesenchyme of keratocystic odontogenic tumor and ameloblastoma was significantly higher than dentigerous and radicular cysts ${ }^{19}$.

Moreover, the results of De Andrade Santos et al showed no significant difference for the expression of matrix metalloproteinase- 9 inthe epithelium of keratocystic odontogenic tumors, and dentigerous and radicular cysts. However, the expression of matrix metalloproteinase-9 inkeratocystic odontogenic epithelial cells was significantly higher than that of dentigerous cyst.37 The findings of Gheisari et al showed that expression of matrix metalloproteinase- 9 in the stroma of dentigerous cysts was significantly lower than keratocystic odontogenic tumors ${ }^{38}$.

In the present study, the expression of matrix metalloproteinase-9 in the epithelium of $54.5 \%$ of dentigerous cysts and $63.2 \%$ of keratocystic odontogenic tumors showed the score 1 . The expression of matrix metalloproteinase-9 in the mesenchyme of $72.7 \%$ of dentigerous cysts and $63.2 \%$ of keratocystic odontogenic tumors showed the score 1 , but in $21.2 \%$ of keratocystic odontogenic tumors, score 2 was observed, whereas none of the dentigerous cysts showed score 2.

Since matrix metalloproteinase-9 plays a major role in the destruction of collagen IV and 
basement membrane, it can be concluded that the increased expression of matrix metalloproteinase9 in keratocystic odontogenic tumor brings about inconsistency and low thickness of collagen IV in this lesion. Inconsistency and low thickness of collagen IV in the basement membrane of keratocystic odontogenic tumor can result in an interaction in the performance of epitheliummesenchyme, which can in turn be considered a factor for the increased development, invasive potential and higher recurrence (different biological behaviors) of keratocystic odontogenic tumors than dentigerous cysts.

\section{REFERENCES}

1. Philipsen HP. Keratocystic odontogenic tumour. Barnes L, EvesonJW, Reichart P, Sidransky D, editors. World Health Organizationclassification of tumors. Pathology and genetics of headand neck tumours. Lyon, France: IARC Press, pp. 306-7.

2. Kolár Z, Geierová M, Bouchal J, Pazdera J, Zboril V, Tvrdý P.Immunohistochemical analysis of the biological potential ofodontogenic keratocysts. J Oral Pathol Med 2006;35:75-80.

3. Da Silva TA, Batista AC, Mendonça EF, Leles CR, Fukada S, Cunha FQ. Comparative expression of Rank, Rankl, andOPG in keratocystic odontogenic tumors, ameloblastomas, anddentigerous cysts. Oral Surg Oral Med Oral Pathol Oral RadiolEndod 2008; 105: 333-41.

4. Oliveira MDC, Souza LB, Pinto LP, Freitas RA. Immunohistochemicalstudy of components of the basement membrane inodontogenic cysts. Pesqui Odontol Bras 2002;16:157-62.

5. Silveira EJD, Piva MR, Galvão HC, Souza LB, Freitas RA. Roleof matrix metalloproteinases in the etiopathogeny of odontogeniccysts. J Bras Patol Med Lab 2007;43:203-9.

6. Freitas VS, Araújo CRF, Alves PM, Souza LB, Galvão HC,Freitas RA. Immunohistochemical expression of matrilysins (MMP-7 and MMP26) in ameloblastomas and adenomatoidodontogenic tumors. Oral Surg Oral Med Oral Pathol Oral Radiol Endod 2009; 108: 417-24.

7. Rosenthal EL, Matrisian LM. Matrix metalloproteases in headand neck cancer. Head Neck 2006;28:639-48.

8. Birkedal-Hansen H, Moore WG, Bodden MK, et al. Matrixmetalloproteinases: a review. Crit Rev Oral Biol Med 1993; 4:197-250.

9. Nabeshima K, Inoue T, Shimao Y, Sameshima T.
Matrixmetalloproteinases in tumor invasion: role for cell migration.Pathol Int 2002; 52: 255-64.

10. Andersen TL, del Carmen Ovejero M, Kirkegaard T,Lenhard T, Foged NT, Delaisse' JM. A scrutiny of matrixmetalloproteinases in osteoclasts: evidence for heterogeneitand for the presence of MMPs synthesized by othercells. Bone 2004; 35: 1107-19

11. Bauvois B. New facets of matrix metalloproteinases MMP-2and MMP-9 as cell surface transducers: outside-in signalingand relationship to tumor progression. Biochem Biophys Acta 2012; 1825: 29-36.

12. Rodriguez D, Morrison CJ, Overall CM. Matrix metalloproteinases:what do they not do? New substrates and biologicalroles identified by murine models and proteomics. BiochimBiophys Acta 2010; 1803: 39-54.

13. Nagatsuka H, Siar CH, Nakano K, et al. Differential expression of collagen IV á1 to á6 chains in basement membranes of benign and malignant odontogenic tumours. Virchows Arch. 2002; 441: 392-99.

14. Tanjore H, Kalluri R. The role of Type IV Collagen and basement membranes in cancer progression and metastasis. Am J Pathol. 2006; 168(3): 715-77.

15. Alaeddini M, Salah S, Dehghan F, Eshghyar N, Etemad-MoghadamS. Comparison of angiogenesis in keratocystic odontogenictumours, dentigerous cysts and ameloblastomas. Oral Dis 2009;15:422-7.

16. Barnes L, Eveson JW, Reichart PA, Sidransky B, editors. Lyon: IARC; 2005. World Health Organization classification of tumors: Pathology and genetics of tumors of the head and neck.

17. Reichart PA, Philipsen HP, Sonner S. Ameloblastoma: Biological profile of 3677 cases. Eur J Cancer B Oral Oncol. 1995; 31B: 86-99. 18.

18. Brannon RB. The odontogenic keratocyst: A clinico-pathologic study of 312 cases. Part II: Histologic features. Oral Surg Oral Med Oral Pathol. 1977; 43: 233-55.

19. Gomes Henriques AC,Galvo HC ,Freitas RA.comprative analysis of the immunohisto chemical expression of collagen IV,mmp9 and TIMP-2 in odontogenic cyst and tumor. Oral Surg Oral Med Oral pathology Oral Radiol Endod 2011;112(4):468-475

20. Gong YWL.the expression of NF-KB,Ki-67, and MMP-9 in CCOT,DGCT and GCOC. Oral Oncol 2009;45(6): 515-520

21. Scariot R,Morosini IC,Torres-Pereira CC,JM Cespedes Amenabar, Barbosa Rebellato NL,Gugisch RC. Immunohistochemical Analysis 
of Metalloproteases in Dentigerous Cysts, Radicular Cysts and KeratocysticOdontogenic Tumors: Systematic Review. Stomatos, 2012; 18(34): 5-15

22. Kubota Y, Ninomiya T, Oka S, Takenoshita Y, Shirasuna K. Interleukin-1 alphadependent regulation of matrix metalloproteinase-9 (MMP9) secretion and activation in the epithelial cells of odontogenic jaw cysts. J Dent Res. 2000, 79(6): 1423-1430.

23. Suojanen J, Lehtonen N, Färkkilä E, Hietanen J, Teronen O, Sorsa T, Hagström J. CommonMatrixMetalloproteinases (MMP-8, 9, -25, and -26) Cannot Explain Dentigerous Cyst Expansion. J Clin Diagn Res. 2014; 8(9): ZC82-5.

24. Kalluri R1.Basement membranes: structure, assembly and role in tumourangiogenesis.Nat Rev Cancer. 2003; 3(6): 422-33

25. Ninomiya Y, Kagawa M, Iyama K, Naito I, Kishiro Y, Seyer JM, Sugimoto M, Oohashi T, Sado Y. Differential expression of two basement membrane collagen genes, COL4A6 and COL4A5, demonstrated by immunofluorescence staining using peptidespecific monoclonal antibodies. J Cell Biol. 1995;130:1219-1229.

26. Nakano S, Iyama K, Ogawa M, Yoshioka H, Sado Y, Oohashi T, Ninomiya Y. Differential tissular expression and localization of type IV collagen $\alpha 1(\mathrm{IV}), \alpha 2(\mathrm{IV}), \alpha 5(\mathrm{IV})$ and $\alpha 6(\mathrm{IV})$ chains and their mRNA in normal breast and in benign and malignant breast tumors. Lab Invest. 1999;79:281-292.

27. Tanaka K, Iyama K, Kitaoka M, Ninomiya Y, Oohashi T, Sado Y, Ono T. Differential expression of $\alpha 1(\mathrm{IV}), \alpha 2(\mathrm{IV}), \alpha 5(\mathrm{IV})$ and $\alpha 6$ (IV) collagen chains in the basement membrane of basal cell carcinoma. Histochem J. 1997; 29:563-570

28. Kiyofuji MA, Iyama K, Kitaoka M, Sado Y, Ninomiya Y, Ueda S. Quantitative analysis of type IV collagen $\alpha$ chains in the basement membrane of human urogenital epithelium. Histochem J. 2002; 34:479-48

29. Hiki Y, Iyama K, Tsuruta J, Egami H, Kamio T, Suko S, Naito I, Sado Y, Ninomiya Y, Ogawa M. Differential distribution of basement membrane type IV collagen $\alpha 1(\mathrm{IV}), \alpha 2(\mathrm{IV}), \alpha 5(\mathrm{IV})$ and $\alpha 6(I V)$ chains in colorectal epithelial tumors. Pathol Int. 2002;52:224-233

30. Nakano S, Iyama K, Ogawa M, Yoshioka H,
Sado Y, Oohashi T, Ninomiya Y. Differential tissular expression and localization of type IV collagen $\alpha 1(\mathrm{IV}), \alpha 2(\mathrm{IV}), \alpha 5(\mathrm{IV})$ and $\alpha 6(\mathrm{IV})$ chains and their mRNA in normal breast and in benign and malignant breast tumors. Lab Invest. 1999;79:281-292.

31. Kadono G, Ishihara T, Yamaguchi T, Kato K, Kondo F, Naito I, Sado Y, Saisho H. Immunohistochemical localization of type IV collagen á chains in the basement membrane of the pancreatic duct in human normal pancreas and pancreatic diseases. Pancreas. 2004; 29: 6166

32. Dehan P, Waltregny D, Beschin A, Noel A, Castronovo V, Tryggvason K, De Leval J, Foidart JM. Loss of type IV collagen $\alpha 5$ and $\alpha 6$ chains in human invasive prostate carcinomas. Am J Pathol. 1997;151:1097-1104.

33. Koei Ikeda, Ken-ichi Iyama, Nobuyuki Ishikawa, Hiroshi Egami, Mitsuyoshi Nakao, Yoshikazu Sado, Yoshifumi Ninomiya, Hideo Baba.Loss of Expression of Type IV Collagen $\alpha 5$ and $\alpha 6$ Chains in Colorectal Cancer Associated with the Hypermethylation of Their Promoter Region Am J Pathol. 2006; 168(3): 856-865.

34. Kannan S, Balaram P, Chandran GJ, Pillai MR, Mathew B,Nalinakumari KR, et al. Alterations in expression of basementmembrane proteins during tumor progression in oral mucosa. Histopathology 1994; 24: 531-7.

35. Tsubura A, Shikata N, Inui T, Morii S, Hatano $\mathrm{T}$, Oikawa $\mathrm{T}$, etal. Immunohistochemical localization of myoepithelial cells andbasement membrane in normal, benign and malignant humanbreast lesions. Virchows Arch 1998; 413: 133-9

36. Poomsawat S,Punyasingh j,Weerapradist W, Expression of basement membrane component in odontogenic cyst.oral Dis 2006; 12(3):290-6

37. De Andrade Santos PP, , de Aquino AR, Oliveira Barreto A, de Almeida Freitas R, Galvão HC, de Souza LB. Immunohistochemical expression of nuclear factor $\kappa \mathrm{B}$, matrix metalloproteinase 9 , and endoglin (CD105) in odontogenic keratocysts, dentigerous cysts, and radicular cysts. Oral Surg Oral Med Oral Pathol Oral Radiol Endod 2011;112(4):476-483

38. Gheisari R,Andishe tadbir A,Dehghan R.matrix metalloproteinase9 expression in dentigerous cyst,odontogenickeratocyst and ameloblastoma. middle-east journal of scientific research 2013;16(6):756-761. 